\title{
Changes of Tears MCP-1 Level After External Dacryocystorhinostomy in Primary Acquired Nasolacrimal Duct Obstruction
}

Müjdat Karabulut ( $\square$ mujdatkarabulut@gmail.com )

Mugla Sitki Kocman Universitesi Tip Fakultesi https://orcid.org/0000-0002-7844-5638

Ercan Saruhan

Mugla Sitki Kocman Universitesi Tip Fakultesi

\section{Research Article}

Keywords: Dacryocystorhinostomy, nasolacrimal duct, monocyte chemoattractant protein, primary acquired nasolacrimal duct obstruction, tears.

Posted Date: November 16th, 2021

DOI: https://doi.org/10.21203/rs.3.rs-1037263/v1

License: (c) (i) This work is licensed under a Creative Commons Attribution 4.0 International License.

Read Full License 


\section{Abstract}

Purpose: To define tears MCP-1 changes after uncomplicated external dacryocystorhinostomy with bicanalicular silicone tube intubation surgery.

Methods: In this prospective, longitudinal study, we included patients diagnosed with primary acquired nasolacrimal duct obstruction and underwent dacryocystorhinostomy with bicanalicular silicone tube intubation surgery. Tears samples were collected with a Schirmer strip placed lateral conjunctival cul-desac and stored in Eppendorf tubes at $-80^{\circ} \mathrm{C}$. At the end of the study, they were taken out of the $-80^{\circ} \mathrm{C}$ cabinet and brought to room temperature. The papers were cut into small pieces and incubated in 2 milliliters tubes with phosphate-buffered saline solution in a shaker at 100 revolutions per minute. Then they were centrifuged at 1.000 revolutions per minute for 5 minutes. MCP- 1 levels were determined by using an ELISA kit.

Results: Of the patients, 14 (56\%) were female, and 11 (44\%) were male. The mean age was $52.7 \pm 10.3$ years (ranged from 30 to 63 years). The MCP-1 levels were $498.66 \pm 101.35 \mathrm{ng} / \mathrm{L}, 576.40 \pm 149.78 \mathrm{ng} / \mathrm{L}$, $422.53 \pm 85.94 \mathrm{ng} / \mathrm{L}, 436.96 \pm 81.38 \mathrm{ng} / \mathrm{L}$ before surgery, in the first week, the first and third months after surgery, respectively. The mean MCP-1 level significantly increased in the first week compared to the preoperative level $(p<0.001)$. In the postoperative first month, there was a prominent decrease $(p<$ 0.001). In the third postoperative month, the mean MCP-1 level was not significantly increased compared to the postoperative first month $(p=0.196)$.

Conclusion: The tears MCP-1 level was significantly decreased after uncomplicated external dacryocystorhinostomy with bicanalicular silicone tube intubation surgery

\section{Introduction}

Primary acquired nasolacrimal duct obstruction (PANDO) arises from idiopathic nasolacrimal duct (NLD) inflammation that results in obstructive fibrosis of the lumen not accompanied by secondary causes such as neoplastic, traumatic, or mechanical origins. External, endoscopic, and endonasal laser dacryocystorhinostomy (DCR) with or without silicon stents have been commonly used surgical techniques to treat PANDO with a variant success rate [1, 2]. External DCR (ext-DCR) provides direct visualization of the surgical area without requiring additional devices such as flexible or rigid nasal endoscopies. A meta-analysis showed that silicon tube intubation did not enhance postoperative complications such as granulation, infection, and adhesion [3]. Moreover, it prevents the ostium's occlusion and increases the surgery's success rate $[3,4]$.

In etiology, various factors such as infections, hormones, tears integrity, drugs, and anatomical variation of adjacent structures have been proposed [5-8]. Studies showed some changes in tears inflammatory (interleukin [IL]-1-2-6), anti-inflammatory (IL-10) cytokines, and growth factors to clarify the biological pathways of the etiology [9]. 
Monocyte chemoattractant protein-1 (MCP-1) is a particular type of cytokine and an effective chemoattractant that has a primary role in monocytes and macrophages migrations to the inflammation sides.[10] As we know, there has been no study revealing the tears MCP-1 changes after any type of DCR in PANDO. This prospective, longitudinal study determined changes in tears MCP-1 level after uncomplicated ext-DCR with bicanalicular silicone tube intubation (BCSI) in PANDO.

\section{Method}

In this prospective, longitudinal study, we included the patients who applied to the ophthalmology clinic with epiphora, were diagnosed as PANDO, and underwent ext-DCR with BCSI in one or both eyes between December 2019 and February 2021. All the patients had epiphora symptoms for at least six months when they enrolled in the study. PANDO was diagnosed performing lacrimal irrigation with a 27-gauge cannula through the dilated upper and lower punctum without flowing the isotonic saline to the throat or nose.

Patients were carefully examined with a biomicroscopy and nasal endoscopy to rule out pathologies of nasal, lacrimal, and adjacent tissues. Patients with congenital or secondary acquired nasolacrimal duct obstruction caused by nasal and paranasal pathologies (septal deviation, nasal cavity masses, sinüsitis, etc.), primary or metastatic lacrimal system tumors, naso-orbital fractures, mechanical reasons (e.g., dacryoliths), active anterior segment and lacrimal system infective-inflammatory pathologies (dacryocystitis, dacryoadenitis, conjunctivitis, keratitis, etc.), eyelid-eyelash pathologies (e.g., entropion, ectropion, distichiasis, trichiasis), history of ophthalmic surgery in the last three months and surgery at any level of the lacrimal drainage system, systemic autoimmune diseases (sarcoidosis, Sjogren disease, Wegener's granulomatosis, etc.), and had been using topical-systemic steroids, immunosuppressiveimmunomodulatory or antiglaucoma drugs were excluded.

All the operations were undertaken by a single surgeon (MK) under general anesthesia. On the operated side, lignocaine and adrenaline-soaked gauze were applied through the nostril to the lateral nasal mucosa to minimize bleeding. Following a $12 \mathrm{~mm}$ vertical skin incision below the medial canthal ligament, the orbicular muscle was split with a monopolar cautery with the help of vein retractors until the periosteum was reached. The periosteum was incised and dissected to expose the lacrimal sac. After opening for about 10-15 mm bone hole, the nasal mucosa was exposed. The nasal gauze was taken out, and the "H shaped" incision was applied to the nasal mucosa and lacrimal sac to create flaps. Posterior flap suturing, bicanalicular silicon tube intubation, and anterior flap suturing were performed, respectively. All the tissues were closed by suturing in layers. The silicon tube was freely released to the nasal cavity after forming a square knot. For the incision side, tobramycin \% 0.3 ointments (Tobrased opt. pomade, Bilim Pharmaceuticals. Inc., Istanbul, Turkey) three times a day for one week was prescribed. Patients also received a combination of loteprednol etabonate $0.5 \%$ and tobramycin $0.3 \%$ opt. susp. (Zylet, Bausch \& Lomb Inc., Rochester, NY), and mometasone furoate \%0.05 nasal spray (Nazoster, Santa Pharma Inc., Istanbul, Turkey) four times a day for two weeks. The skin sutures were removed during the first postoperative week. The silicon tubes stayed at least four months. 
Tears samples were collected one day before surgery, in the first week, the first, and the third month after surgery. Tears samples were collected with a Schirmer strip that was placed lateral conjunctival cul-desac for about five minutes until at least $15 \mathrm{~mm}$ of the strip was filled with tears. The amount of tears was calculated in microliters with a regression graph corresponding to the Schirmer strips' tears level. The samples were stored in eppendorf tubes at $-80^{\circ} \mathrm{C}$ within 10 minutes after sampling.

At the end of the study, the samples were taken out of the $-80^{\circ} \mathrm{C}$ cabinet and brought to room temperature. The papers were cut into small pieces and incubated in 2 milliliters $(\mathrm{mL})$ tubes with phosphate-buffered saline solution at room temperature in a shaker at 100 revolutions per minute (rpm) for about three hours. Then they were centrifuged at $1.000 \mathrm{rpm}$ for five minutes. MCP-1 concentrations were measured in tears using human-specific enzyme-linked immunosorbent assays (ELISA) (BTlaboratory, Shanghai, China) according to the manufacturer's instructions. MCP-1 assay sensitivity was $2.43 \mathrm{ng} / \mathrm{L}$ with inter-assay and intra-assay coefficients of variation less than $10 \%$ and $8 \%$, respectively. The statistical program recorded the data and represented the mean \pm standard deviation for continuous variables and frequencies (percentages) for categorical variables. Friedman's analysis of variance and Wilcoxon tests were used for repeated measures analysis and pairwise comparisons. $P<0.05$ was defined as statistically significant. Power analysis was performed using GPower 3.1 software.

Muğla Sitki Koçman University Clinical Research Ethics Committee approved this study with a 06/VI decision number. All patients provided informed consent. The Declaration of Helsinki's principle was consistently followed throughout the study.

\section{Results}

Thirty-two eyes of 30 patients who met the inclusion criteria were included. However, three eyes of three patients who did not attend at least one of three postoperative visits and two eyes of two patients with recurrent obstruction were excluded. Post hoc power calculations were applied, and the sample size was seen to provide 0.989 power and 0.808 effects size for MCP- 1 at a error probability level of 0.05 . The silicone tubes were in place in all eyes in the third month of the surgery. None of the remaining patients developed postoperative complications such as wound infection, dehiscence, silicon tube prolapse, rhinostomy fibrosis, and intranasal synechiae. Of the patients, 14 (56\%) were female, and $11(44 \%)$ were male. The mean age was $52.7 \pm 10.3$ years (ranged from 30 to 63 years) (Table 1). 
Table 1

Demographic characteristics of the patients.

\begin{tabular}{llll} 
Ext-DCR with BCSI & Eyes/ patients $(\mathrm{n})$ & $\begin{array}{l}\text { Age (year) } \\
\text { Mean } \pm \text { SD }\end{array}$ & Gender (n, \%) \\
\cline { 2 - 4 } & & $52.7 \pm 10.3$ & $14(56 \%) / 11(44 \%)$ \\
& (range, 30 to 63) &
\end{tabular}

Abbreviations: BCSI, bicanalicular silicone tube intubation; ext-DCR, external dacryocystorhinostomy; $\mathrm{F}$, female; $\mathrm{M}$, male; $\mathrm{n}$, number; SD, standard deviation.

The MCP-1 levels were $498.66 \pm 101.35$ nanograms per liter (ng/L), $576.40 \pm 149.78$ ng/L, $422.53 \pm 85.94$ $\mathrm{ng} / \mathrm{L}, 436.96 \pm 81.38 \mathrm{ng} / \mathrm{L}$ before surgery, in the first week, the first and third months after surgery, respectively. There was a significant difference among these values $(p<0.001)($ Table 2$)$.

Table 2

Changes in MCP-1 levels in the postoperative periods.

Preoperative

(baseline)

Mean \pm SD

MCP-1 levels

(ng/L)
Postoperative

Mean \pm SD

P-value

1st week

$576.40 \pm 149.78^{b}$ 1st month

$422.53 \pm 85.94^{c}$ 3rd month

$498.66 \pm 101.35^{a} \quad 576.40 \pm 149.78^{b} \quad 422.53 \pm 85.94^{c} \quad 436.96 \pm 81.38^{c} \quad<0.001^{*}$

Abbreviations: MCP-1, monocyte chemoattractant protein-1; ng/L, nanograms per liter, SD, standard deviation.

* Repeated measures analysis with Friedman's analysis of variance showed significant differences among the values.

$a, b, c$ The same and different letters indicate the similar and significantly different values in pairwise comparisons with the Wilcoxon test.

The mean MCP-1 level significantly increased in the first week compared to the preoperative level $(p<$ 0.001). There was a prominent decrease in the postoperative first month compared to the preoperative level $(p<0.001)$. In the third postoperative month, the mean MCP-1 level was not significantly increased compared to the postoperative first month $(p=0.196)$ (Figure 1).

\section{Discussion}

This prospective, longitudinal study showed that the tears MCP-1 level significantly increased in the postoperative first week and had a prominent decrease in the postoperative first month compared to the preoperative level after uncomplicated ext-DCR with BCSI surgery. Compared to the postoperative first month, it was not significantly changed in the postoperative third month, and its low level was continued. 
The etiopathogenesis of PANDO has not been well understood yet. On the other hand, changes in tears proteins and electrolytes, dysregulation of some biological pathways in NLD epithelium, such as increased inflammation, cellular proliferation, and decreased apoptosis, have been thought to be associated with NLD lumen obstruction $[7,9,11,12]$.

In some studies, the alterations of the tears inflammatory and anti-inflammatory cytokines and growth factors have been demonstrated to clarify the etiopathogenesis of PANDO. Ali MJ et al. reported upregulation of 10 pro-inflammatory and three anti-inflammatory cytokines in tears of eyes with PANDO compared to the healthy fellow and control eyes [7]. In a comparative study, Lee JK et al. demonstrated that IL-1, IL-6, IL-10, transforming growth factor- $\beta 2$, fibroblast growth factor-2, vascular endothelial growth factor significantly increased in eyes with PANDO compared to the normal eyes, and a significant decrease was shown in these cytokines after endoscopic DCR.[9] Andalib D et al. showed in a casecontrol study that IL-1 $\beta$ level was predominantly increased in eyes with PANDO in comparison with the healthy fellow and control eyes [13].

Acute inflammation begins fast and proceeds a few days or weeks, whereas chronic inflammation lasts for months or years. MCP-1 is a cytokine that regulates monocyte migration and infiltration in the inflamed area in acute and chronic inflammations [14]. Increased expression of MCP-1 was reported in vitreous samples of the eyes with retinal detachment, diabetic retinopathy, and in aqueous humor samples of eyes after phacoemulsification surgery [15-18]. Since pterygium and dry eye syndrome were chronic inflammatory disorders, it was thought that high expression of MCP-1 might have a role in the etiopathogenesis of these diseases [19]. Additionally, in patients with Stevens-Johnson syndrome, dramatically increased tears MCP-1 level was demonstrated [20, 21].

To the best of our knowledge, there has been no study indicating the tears MCP-1 level changes after extDCR with BSTI in PANDO. Our study found a significant increase in the tears MCP-1 level in the first week compared to the baseline. We thought this increase could be originated from the inflammation and proliferation stage of the wound healing process. In the first month, its level was dramatically decreased compared to the first week and baseline. Besides, the lower level of MCP-1 continued and was not significantly changed in the third month compared to the first month. Our patients were prescribed topical loteprednol etabonate $0.5 \%$ and intranasal mometasone furoate $\% 0.05$ spray four times a day for two weeks. Topical and intranasal steroids could accelerate the decrease of tears MCP-1 level. However, these steroids have a short half-life and are eliminated rapidly [22, 23]. Therefore, administration of these steroids for two weeks after surgery could not have reduced the tears MCP- 1 level in the first and third months. So we thought that the decrease of the tears MCP-1 level in the first and third months was not related to two weeks administration of these steroids. Instead, this dramatic decrease in the first month and stable duration might be related to resolving of the tears drainage failure. Previous studies have supported our hypothesis by indicating that tears clearance insufficiency may induce inflammatory cytokines due to ocular surface irritation [24, 25]. 
This study was limited in some ways. The duration of the study was relatively short, and the silicone stents were not removed at the end of the study. Extubation of the silicon tubes and long study duration might have affected the results. Besides, we only showed the changes of the tears MCP-1 level after extDCR with BCSI in PANDO. We did not compare it with the tears of fellow eyes and age, sex-matched healthy controls.

In conclusion, the tears MCP-1 level was significantly decreased after ext-DCR with BCSI in PANDO. These results might be interpreted as that uncomplicated ext-DCR with $\mathrm{BSCl}$ reduces inflammation by enhancing tears flow, clearance, and relieving ocular irritations. Prospective studies with a prolonged follow-up are needed to demonstrate this surgery's long-term effects on tears cytokine levels.

\section{Declarations}

Ethics approval and consent to participate: We followed the local institutional research committee's ethical standards and the 1964 Helsinki declaration of ethical standards in this study's procedures. Informed consent was gathered from all participants. Muğla Sıtkı Koçman University Clinical Research Ethics Committee approved this study.

Consent for publication: Not applicable

Availability of data and materials: All data are included in this published article.

Competing interests: The authors listed above have no conflicts of interest to declare.

Funding: This research did not take any specific grant from funding agencies in the public, commercial, or other sectors.

Authors' contributions: Müjdat Karabulut, Ercan Saruhan, have made substantial contributions to conception and design, data acquisition, analysis, and data interpretation. All authors have given final approval of the version to be published. All the authors agree to be accountable for all aspects of the work in ensuring that questions related to the accuracy or integrity of any part of the work are appropriately investigated and resolved.

\section{Acknowledgments}

Not applicable

\section{References}

1. Zengin M, Eren $E$ (2014) The return of the jedi: comparison of the outcomes of endolaser dacryocystorhinostomy and endonasal dacryocystorhinostomy. Int Forum Allergy Rhinol 4(6):480483 
2. Sobel RK, Aakalu VK, Wladis EJ, Bilyk JR, Yen MT, Mawn LA (2019) A Comparison of endonasal dacryocystorhinostomy and external dacryocystorhinostomy: A report by the American Academy of Ophthalmology. Ophthalmology 126(11):1580-1585

3. Xie C, Zhang L, Liu Y, Ma H, Li S (2017) Comparing the success rate of dacryocystorhinostomy with and without silicone intubation: A trial sequential analysis of randomized control trials. Sci Rep 7(1):1936

4. Yakopson VS, Flanagan JC, Ahn D, Luo BP (2011) Dacryocystorhinostomy: History, evolution and future directions. Saudi J Ophthalmol 25(1):37-49

5. Ali MJ, Paulsen F (2019) Etiopathogenesis of primary acquired nasolacrimal duct obstruction: what we know and what we need to know. Ophthalmic Plast Reconstr Surg 35(5):426-433

6. Kamal S, Ali M (2015) Primary acquired nasolacrimal duct obstruction (PANDO) and secondary acquired lacrimal duct obstructions (SALDO). In., edn.;:133-142

7. Ali MJ, Patnaik S, Kelkar N, Ali MH, Kaur I (2020) Alteration of tear cytokine expressions in primary acquired nasolacrimal duct obstruction - Potential insights into the etiopathogenesis. Curr Eye Res 45(4):435-439

8. Liu Y, Zhang Z, Jin Q, Liu Y, Kang Z, Huo Y et al (2019) Hyperprolactinemia is associated with a high prevalence of serum autoantibodies, high levels of inflammatory cytokines and an abnormal distribution of peripheral B-cell subsets. Endocrine 64(3):648-656

9. Lee JK, Kim TH (2014) Changes in cytokines in tears after endoscopic endonasal dacryocystorhinostomy for primary acquired nasolacrimal duct obstruction. Eye (Lond) 28(5):600607

10. Melgarejo E, Medina MA, Sánchez-Jiménez F, Urdiales JL (2009) Monocyte chemoattractant protein1: a key mediator in inflammatory processes. Int J Biochem Cell Biol 41(5):998-1001

11. Lew H, Lee SY, Yun YS (2004) Measurement of pH, electrolytes and electrophoretic studies of tear proteins in tears of patients with dacryoliths: a novel concept for dacryoliths. Ophthalmologica 218(2):130-135

12. Paulsen FP, Thale AB, Maune S, Tillmann BN (2001) New insights into the pathophysiology of primary acquired dacryostenosis. Ophthalmology 108(12):2329-2336

13. Andalib D, Ghorbani Haghjoo A, Salimi M, Najafzadeh F, Shahbazi A (2016) Comparison of IL-1 $\beta$ concentration of tear between cases with primary acquired nasolacrimal duct obstruction and cases without nasolacrimal duct obstruction. Bina J Ophthalmol 21(4):316-321

14. Deshmane SL, Kremlev S, Amini S, Sawaya BE (2009) Monocyte chemoattractant protein-1 (MCP-1): an overview. J Interferon Cytokine Res 29(6):313-326

15. Nakazawa T, Hisatomi T, Nakazawa C, Noda K, Maruyama K, She H et al (2007) Monocyte chemoattractant protein 1 mediates retinal detachment-induced photoreceptor apoptosis. Proc Natl Acad Sci U S A 104(7):2425-2430

16. Fonollosa A, Garcia-Arumi J, Santos E, Macia C, Fernandez P, Segura RM et al (2010) Vitreous levels of interleukine-8 and monocyte chemoattractant protein-1 in macular oedema with branch retinal 
vein occlusion. Eye 24(7):1284-1290

17. Kawai M, Inoue T, Inatani M, Tsuboi N, Shobayashi K, Matsukawa A et al (2012) Elevated levels of monocyte chemoattractant protein-1 in the aqueous humor after phacoemulsification. Invest Ophthalmol Vis Sci 53(13):7951-7960

18. Yoshida S, Kobayashi Y, Nakao S, Sassa Y, Hisatomi T, Ikeda Y et al (2017) Differential association of elevated inflammatory cytokines with postoperative fibrous proliferation and neovascularization after unsuccessful vitrectomy in eyes with proliferative diabetic retinopathy. Clin Ophthalmol 11:1697-1705

19. Liu C, Song Y, Wang X, Lai Z, Li C, Wan P et al (2020) The key role of VEGF in the cross talk between pterygium and dry eye and its clinical significance. Ophthalmic Res 63(3):320-331

20. Yagi T, Sotozono C, Tanaka M, Fuwa M, Sekiyama E, Ueta M et al (2011) Cytokine storm arising on the ocular surface in a patient with Stevens-Johnson syndrome. $\mathrm{Br} J$ Ophthalmol 95(7):1030-1031

21. Ueta M, Sotozono C, Yokoi N, Kinoshita S (2012) Downregulation of Monocyte Chemoattractant Protein 1 Expression by Prostaglandin E2 in Human Ocular Surface Epithelium. Arch Ophthalmol 130(2):249-251

22. Amon M, Busin M (2012) Loteprednol etabonate ophthalmic suspension $0.5 \%$ : efficacy and safety for postoperative anti-inflammatory use. Int Ophthalmol 32(5):507-517

23. Patel D, Garadi R, Brubaker M, Conroy JP, Kaji Y, Crenshaw K (2007) Onset and duration of action of nasal sprays in seasonal allergic rhinitis patients: Olopatadine hydrochloride versus mometasone furoate monohydrate. Allergy Asthma Proc 28(5):592-599

24. Prabhasawat $P$, Tseng SC (1998) Frequent association of delayed tear clearance in ocular irritation. $\mathrm{Br} J$ Ophthalmol 82(6):666-675

25. Lindén C, Alm A (1990) The effect of reduced tear drainage on corneal and aqueous concentrations of topically applied fluorescein. Acta Ophthalmol (Copenh) 68(6):633-638

\section{Figures}




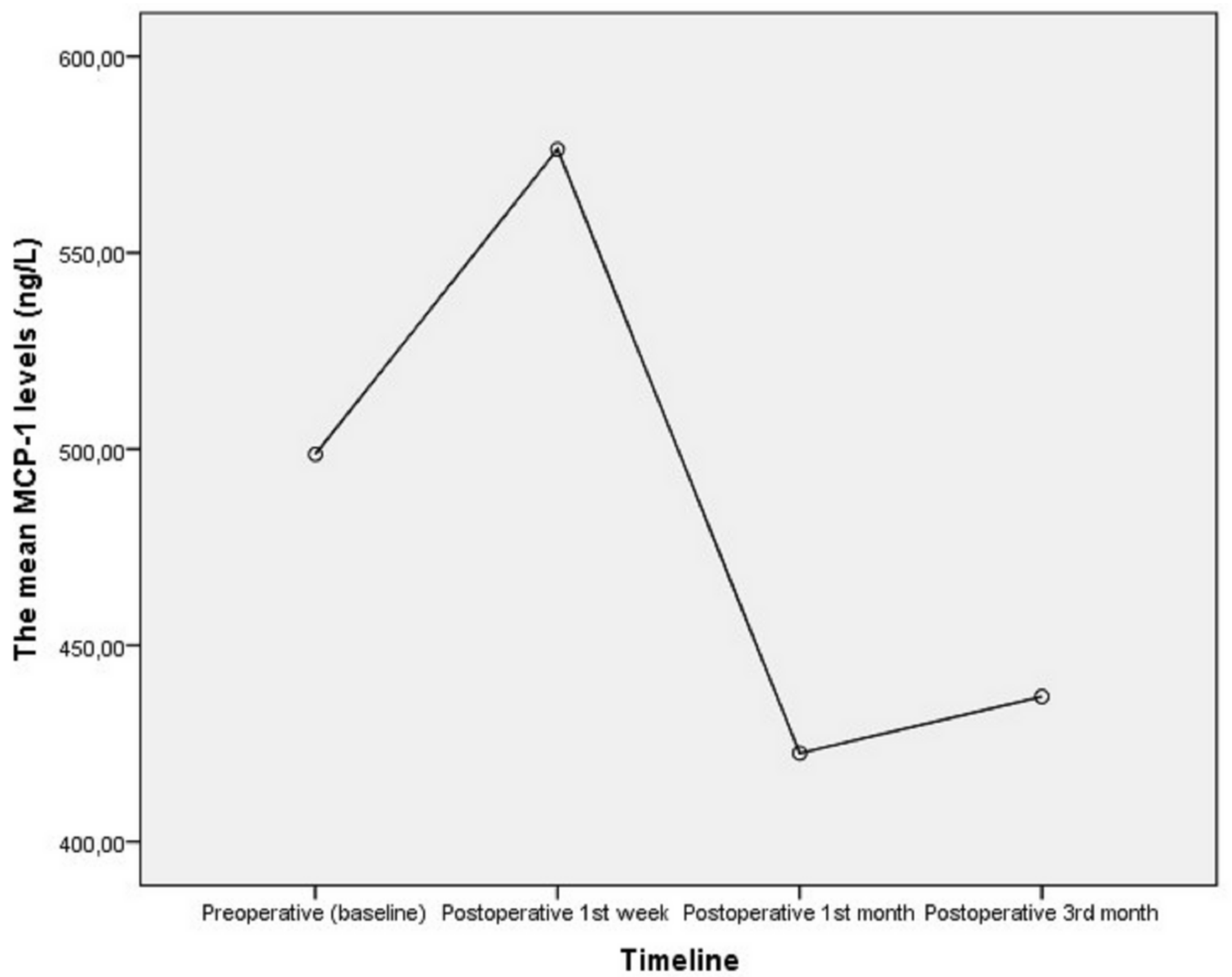

\section{Figure 1}

The tears MCP-1 level changes over time. It was significantly increased in the postoperative first week ( $p<$ $0.001)$, and there was a dramatic decrease in the postoperative first month $(p<0.001)$. The mean MCP-1 level change was not significant in the postoperative third month compared to the first month $(p=0.196)$. 